\title{
Effect of the quality of the cumulus-oocyte complex in the domestic cat on the ability of oocytes to mature, fertilize and develop into blastocysts in vitro
}

\author{
T. C. Wood ${ }^{1,2}$ and D. E. Wildt ${ }^{1}$ \\ ${ }^{1}$ National Zoological Park, Conservation and Research Center, Smithsonian Institution, Washington, \\ DC 20008 and Front Royal, VA 22630, USA; and ${ }^{2}$ Department of Biology, George Mason University, \\ Fairfax, VA 22030, USA
}

\begin{abstract}
Immature cumulus-oocyte complexes (COCs) were recovered from freshly excised domestic cat ovaries and graded at a magnification of $\times 40$ for the condition of the cumulus oophorus of the oocyte cytoplasm. Grade I and II COCs were those with a uniformly dark cytoplasm and a readily identifiable, eccentrically located germinal vesicle. Grade I COCs had five or more cumulus oophorus cell layers, whereas grade II complements had less than five cell layers. Grade III and IV COCs were those undergoing progressive stages of oocyte cytoplasmic deterioration indicated by transparency or mosaic fragmentation and partialto-complete loss of cumulus oophorus cells. In Expt 1, 699 oocytes were cultured for maturation and fertilization in vitro. More $(P<0.05)$ oocytes from grade I COCs matured $(59.3 \%)$ and fertilized $(29.7 \%)$ than from all other grades. Maturation and fertilization success did not differ $(P>0.05)$ for grade II $(32.4,11.6 \%$, respectively) and grade III $(21.9,5.1 \%)$ oocytes, but these values were superior $(P<0.05)$ to those of grade IV $(5.1,1.4 \%)$. In Expt 2, 1040 COCs were graded, cultured for maturation and then inseminated. Of grade I oocytes, $24.4 \%$ developed into blastocysts compared with only $5.3 \%$ of grade II oocytes $(P<0.05)$. In general, oocytes from grade III and IV COCs were incapable of cleaving or growing in vitro. Of the 1739 COCs collected for both experiments, $12.3 \%$ met grade I criteria, the only category that provided consistent maturation, fertilization and development to blastocyst stage in vitro. In summary, a highly heterogeneous population of cumulus-oocyte complexes can be separated in the cat on the basis of grossly apparent morphological characteristics that, in turn, reflect functional differences in the ability of oocytes to mature, fertilize and develop in vitro.
\end{abstract}

\section{Introduction}

Several laboratories have independently assessed the potential of maturing and fertilizing domestic cat oocytes, mainly using in vitro oocyte maturation (IVM) and fertilization (IVF) approaches developed for other mammals (Johnston et al., 1989, 1991a, b, 1993; Goodrowe et al., 1991; Luvoni and Oliva, 1993; Pope et al., 1994, 1995; Wood et al., 1995; Wolfe and Wildt, 1996). The biological competency of IVM/IVF domestic cat embryos has been demonstrated after embryo transfer (Pope et al., 1995). None the less, it has become apparent that IVM/IVF success in the cat is generally less than that reported for other commonly studied species like the cow (Harper and Bracket, 1993; Lonergan et al., 1994; Trounson et al., 1994; Blondin and Sirard, 1995) and mouse (Eppig and O'Brien, 1996). For example, it is not unusual for $60-80 \%$ of cow antral follicular oocytes to be fertilized and to cleave in vitro (Harper and Brackett, 1993; Blondin and Sirard, 1995).

Received 17 January 1997
Techniques in the mouse have progressed even further to allow the growth, maturation and successful fertilization of oocytes from primordial ovarian follicles (Eppig and O'Brien, 1996). In contrast, only about $50-60 \%$ of cultured cat oocytes achieve nuclear maturation in culture and, after insemination, usually $<40 \%$ are fertilized on the basis of embryo cleavage (Johnston et al., 1993; Wood et al., 1995; Wolfe and Wildt, 1996). Even under optimal culture conditions, $<20 \%$ of these cleaved embryos grow into blastocysts in vitro (Wolfe and Wildt, 1996).

We have been interested in the cause(s) for the comparatively low IVM/IVF success rate in cultured oocytes of the domestic cat, a finding that extends to its wild relatives (Johnston et al., 1991c). In some cases (especially attempts to rescue immature intraovarian oocytes from endangered felid species), poor IVM/IVF efficiency is due to suboptimal health or an aged oocyte donor (Johnston et al., 1991c). However, it is now known that the ability of cat oocytes matured in vivo or in vitro to be fertilized can be influenced by a host of other factors, including the pre-culture storage interval and 
maturation time (Wolfe and Wildt, 1996), the culture medium itself (Johnston et al., 1993), protein and hormone supplementation (Johnston et al., 1991a; Wood et al., 1995) and the temperature or gas environment (Johnston et al., 1991b). We have considered low oocyte maturation success in the cat in the context of the inherent induced ovulatory mechanism of the species (Greulich, 1934; Wildt et al., 1980). As such, the cat has no means of spontaneously releasing its oocytes. This may explain, in part, why a recent histological study in our laboratory revealed that, at any given time, about $65 \%$ of intraovarian cumulus-oocyte complexes (COCs) are expressing atresia-related changes in the oocyte cytoplasm or cumulus cell complement (Wood et al., 1997). Thus, during routine harvest from whole ovaries, many oocytes are already in the throes of degenerative turnover and are incapable of maturation or fertilization in vitro.

Therefore, it became logical to test the hypothesis that there are readily recognizable morphological characteristics in cat oocytes that reflect overall quality and the ability to mature, to be fertilized and develop to an advanced embryo stage in vitro. On the basis of our recent characterization of atretic events in cat ovarian follicles (Wood et al., 1997), we identified phenotypic traits compatible with developing a simple, objective quality grading system for COCs; and then determined whether COCs meeting different quality criteria varied in functional competency, specifically the ability of an oocyte to mature, be fertilized and develop to an advanced embryo stage in vitro.

\section{Materials and Methods}

\section{Collection and culture of $\mathrm{COCS}$}

Ovaries from sexually mature queens were recovered following routine ovariohysterectomy at local veterinary clinics (Wood et al., 1995, 1997). Briefly, whole ovaries were immediately placed in Dulbecco's phosphate-buffered saline containing $100 \mathrm{iu}$ penicillin $\mathrm{ml}^{-1}$ and $100 \mathrm{iu}$ streptomycin $\mathrm{ml}^{-1}$ (Sigma Chemical Co., St Louis, MO) and maintained at room temperature $\left(22^{\circ} \mathrm{C}\right)$ for $\mathrm{I}-4 \mathrm{~h}$ before oocyte recovery. Oocytes were harvested from ovaries into $60 \mathrm{~mm}$ tissue culture dishes containing minimal essential medium (Sigma) comprising

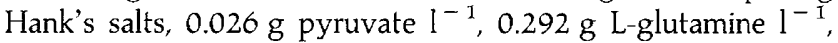
$0.4 \%(\mathrm{w} / \mathrm{v})$ BSA (Sigma), $100 \mathrm{iu}$ penicillin $\mathrm{ml}^{-1}$, 100 iu streptomycin $\mathrm{ml}^{-1}$ and $25 \mathrm{mmol}$ Hepes buffer $\mathrm{l}^{-1}$. Each ovary was sliced longitudinally with a scalpel blade followed by lateral mincing of the ovarian cortex. Collected oocytes were graded (see below), gently rinsed in a fresh dish of culture medium and immediately placed in $50 \mu \mathrm{l}$ drops of culture medium under mineral oil (Mallinckrodt Inc., Paris, KY). The culture medium consisted of Eagle's minimum essential medium containing $0.026 \mathrm{~g}$ pyruvate $\mathrm{l}^{-1}, 0.292 \mathrm{~g} \mathrm{~L}$-glutamine $\mathrm{l}^{-1}, 0.4 \%(\mathrm{w} / \mathrm{v})$ BSA (Sigma), $100 \mathrm{iu}$ penicillin $\mathrm{ml}^{-1}, 100 \mathrm{iu}$ streptomycin $\mathrm{ml}^{-1}, 1 \mu \mathrm{g} \mathrm{LH} \mathrm{ml}{ }^{-1}$ (NIADDK-oLH-25; National Hormone

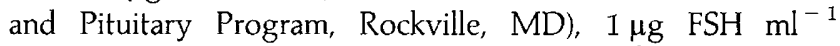
(NIADDK-oFSH-17) and I $\mu \mathrm{g}$ oestradiol $\mathrm{ml}^{-1}$ (Sigma). Both the collection and culture medium were pre-equilibrated in a $38^{\circ} \mathrm{C}$ humidified incubator containing $5 \% \mathrm{CO}_{2}$ in air. Similar incubation conditions were used for all cultures. Oocytes were separated by grade and cultured for $24 \mathrm{~h}$ in groups of 5-25 oocytes per $50 \mu \mathrm{l}$ drop to achieve meiotic maturation before co-culture with spermatozoa (Wood et al., 1995; Wolfe and Wildt, 1996).

\section{Grading criteria for oocytes}

Criteria for grading COCs were based on the condition of the oocyte cytoplasm and the cumulus cell complement, traits that allow clear characterization of four distinctive COC types. The grading system was facilitated by a pre-emptive characterization of the detailed progressive events associated with normal atresia in follicle-oocyte complexes within whole sections of freshly excised cat ovaries (Wood et al., 1997). These earlier observations were used to establish objective grading criteria for COCs with the assistance of a standard stereomicroscope.

\section{Semen collection and in vitro fertilization}

Oocytes cultured for meiotic maturation were co-cultured with normospermic semen (Johnston et al., 1991a; Wood et al., 1995) from a single male donor. Semen was collected with an artificial vagina, centrifuged and subjected to swim-up processing as described by Goodrowe et al. (1989) and Wood et al. (1995). Briefly, freshly collected semen was diluted 1:I (v/v) with Ham's F10 medium (Irvine Scientific, Santa Ana, CA) supplemented with $0.026 \mathrm{~g}$ pyruvate $\mathrm{l}^{-1}, 0.292 \mathrm{~g}$ L-glutamine $\mathrm{l}^{-1}, 100 \mathrm{iu}$ penicillin $\mathrm{ml}^{-1}, 100$ iu streptomycin $\mathrm{ml}^{-1}$ and $5 \%$ (v/v) fetal calf serum (FCS; Hyclone Laboratories, Logan, UT; lot 11152381). Diluted semen was centrifuged ( $300 \mathrm{~g}, 8 \mathrm{~min}$ ) and the supernatant aspirated and discarded; $100 \mu \mathrm{l}$ of fresh Ham's FIO was gently layered onto the remaining sperm pellet. After $1 \mathrm{~h}$ at room temperature $\left(22^{\circ} \mathrm{C}\right)$, the swim-up fraction was removed and sperm concentration, percentage motility and progressive motility status determined (Goodrowe et al., 1989). All swim-up fractions were assessed as having greater than $80 \%$ progressive sperm motility with a status of 3.0 or greater, which is excellent quality for IVF in felids (Goodrowe et al., 1989). An aliquot of the swim-up fraction was diluted with Ham's F10 for a final concentration of $2 \times 10^{5}$ motile spermatozoa $\mathrm{ml}^{-1}$ for the fertilization medium. After the $24 \mathrm{~h}$ culture, oocytes were removed from the $50 \mu \mathrm{l}$ maturation drops and placed in $100 \mu \mathrm{l}$ drops of fertilization medium. After a period of co-culture, oocytes were evaluated for fertilization or cleavage and development depending on the experiment.

\section{Assessment of oocyte maturation, fertilization and embryo development}

Assessment of oocyte maturation and fertilization was conducted after $15 \mathrm{~h}$ of co-culture with spermatozoa. Oocytes were gently pipetted with a small bore pipette to remove cumulus cells. Oocytes then were fixed in $0.3 \%(\mathrm{w} / \mathrm{v})$ glutaraldehyde in Dulbecco's phosphate-buffered saline for $10 \mathrm{~min}$ before staining with $0.5 \mathrm{mg} 4,6$ diamidino-2-phenylindole $\mathrm{ml}^{-1}$ (DAPI; Sigma) in the dark for an additional $10 \mathrm{~min}$ (Wood et al., 1995). Groups of stained oocytes were placed on a microscope slide with a minimal amount of medium $(5 \mu \mathrm{l})$ and Downloaded from Bioscientifica.com at 04/26/2023 10:54:49AM 
covered with a $22 \mathrm{~mm}^{2}$ coverslip. A petroleum jelly:paraffin wax mixture (1:1) was sparingly applied to the edges of the coverslip to allow gentle seating of the coverslip on the oocytes. The coverslip was compressed while observing the oocytes flatten slightly, avoiding rupture, under a dissecting stereomicroscope $(\times 30)$. Chromatin of each oocyte was evaluated immediately under epifluorescence excitation at a magnification of $\times 400$ (Leitz Diaplan microscope, Wetzlar). Any oocyte exhibiting a germinal vesicle, germinal vesicle breakdown, metaphase of meiosis I or degeneration was termed immature. Oocytes in telophase of meiosis I, metaphase of meiosis II or fertilized were considered mature (Johnston $e t$ al., 1989).

Fertilization was based on evidence of at least two pronuclei (Goodrowe et al., 1989; Johnston et al., 1991a). All oocytes initially graded and cultured were included in evaluations. During the embryo culture for 10 days, the culture medium was changed every $48 \mathrm{~h}$. Parthenogenetic controls were assessed in trials in which embryos were allowed to cleave and develop. These oocytes were cultured under similar conditions as inseminated oocytes, but without spermatozoa in the medium.

\section{Experimental design}

For accurate evaluation of maturation and fertilization of graded oocytes, it was necessary to fix oocytes soon after fertilization and before onset of chromatin degeneration. Therefore, the first experiment focused on maturation and fertilization characteristics of graded oocytes fixed after $24 \mathrm{~h}$ of maturation and $15 \mathrm{~h}$ of co-culture with spermatozoa. A second experiment evaluated the ability of oocytes from various grades of COCs to cleave and develop in vitro. All COCs initially graded and cultured for meiotic maturation $(24 \mathrm{~h})$ were evaluated at $24 \mathrm{~h}$ intervals for 10 days after IVF.

\section{Statistical analyses}

For each experiment, the number of oocytes maturing, fertilizing or developing as embryos was expressed as a percentage of total oocytes assigned per treatment. A general loglinear analysis was performed on data from each experiment. When appropriate, Bonferroni $t$ tests (Dunn, 1961) between loglinear parameters for oocyte grade were performed for maturation, fertilization and stages of embryo development.

\section{Results}

\section{COC quality grades}

Four distinct COC grades (I = excellent; II = good; III = fair; IV = poor) were distinguished on the basis of appearance of the oocyte cytoplasm and cumulus cell complement (Fig. 1). This classification scheme was based mainly on our recent histological observations that oocyte cytoplasmic atresia is associated with (i) aggregation of small lipid yolk droplets to form large vacuoles that accumulate centrally and (ii) cytoplasmic fragmentation that is usually portrayed in a translucent, mosaic pattern (Wood et al., 1997). These conditions were readily detectable macroscopically $(\times 40)$ in the cat oocyte. In general, grade I and II oocytes had highly opaque, uniform cytoplasm with readily detectable, round germinal vesicles (Fig. 1a, b). Large aggregations of lipid droplets were associated with mosaic transparency and was typical of grade III COCs (Fig. Ic). More advanced fragmentation of the cytoplasm was associated with progressive degenerative atresia and was, therefore, used to classify lower grade COCs to a score of IV (Fig. Id).

Cumulus cell complements also were considered in the COC grading process. Grade I COCs had more layers of cumulus cells (five or more) than did grade II oocytes (2-4 layers) (Fig. 1a, b). In contrast, grade III COCs were characterized by a complete corona radiata but only partial layers of cumulus oophorus cells (Fig. Ic). Partial-to-near total dissociation of the cumulus cells and the oocyte cytoplasm was characteristic of grade IV COCs and usually appeared as a loose association of the corona radiata and a sparse cumulus oophorus layer (Fig. 1d). However, partially-to-completely denuded oocytes were also included in the grade IV classification.

\section{Experiment 1: oocyte maturation and fertilization}

A total of 699 COCs was harvested (19 ovarian pairs) for grading and for maturation and fertilization competence. Of these, 91 (13.0\%) met grade I criteria, $216(30.9 \%)$ grade II, 178 $(25.5 \%)$ grade III and $214(30.6 \%)$ grade IV (Table 1). Grade I oocytes were superior in their ability to mature and be fertilized compared with all other grades $(P<0.05)$. Nearly twice as many grade I oocytes matured and more than twice of these were fertilized compared with grade II oocytes (Table 1). Although there was a $10 \%$ advantage in maturation and $>6 \%$ advantage in fertilization success for oocytes from grade II compared with grade III COCs, the differences were not significant $(P>0.05)$. Oocytes from grade IV COCs were poor in terms of maturation (compared with grades I to III) and fertilization (compared with grades I and II).

\section{Experiment 2: oocyte cleavage and development}

A total of $1150 \mathrm{COCs}$ was harvested (30 ovarian pairs) to evaluate the ability of oocytes from different $\mathrm{COC}$ grades to cleave and develop as embryos. Of these, 110 COCs were cultured across the four grades as parthenogenetic controls: 24 grade I, 37 grade II, 33 grade III and 16 grade IV. Similar to previous reports (Johnston et al., 1989; Wood et al., 1995; Wolfe and Wildt, 1996), three COCs (one grade I and two grade III) were parthenogenetically activated and cleaved (2.7\%). Of COCs co-cultured with spermatozoa, $123(11.8 \%)$ met grade I criteria, $226(21.7 \%)$ grade II, $296(28.5 \%)$ grade III and $395(38.0 \%)$ grade IV. Oocytes from grade I COCs were superior in their ability to cleave and develop in vitro compared with all other graded categories $(P<0.05$; Table 2$)$. More than $35 \%$ of these oocytes cleaved and $>30.0 \%$ developed to at least the morula stage, with more than 24.0 and $17 \%$ reaching the blastocyst and expanded blastocyst stage, respectively (Wolfe and Wildt, 1996). The kinetics of embryo cleavage were similar to development measured in vivo (Roth et al., 1994; 


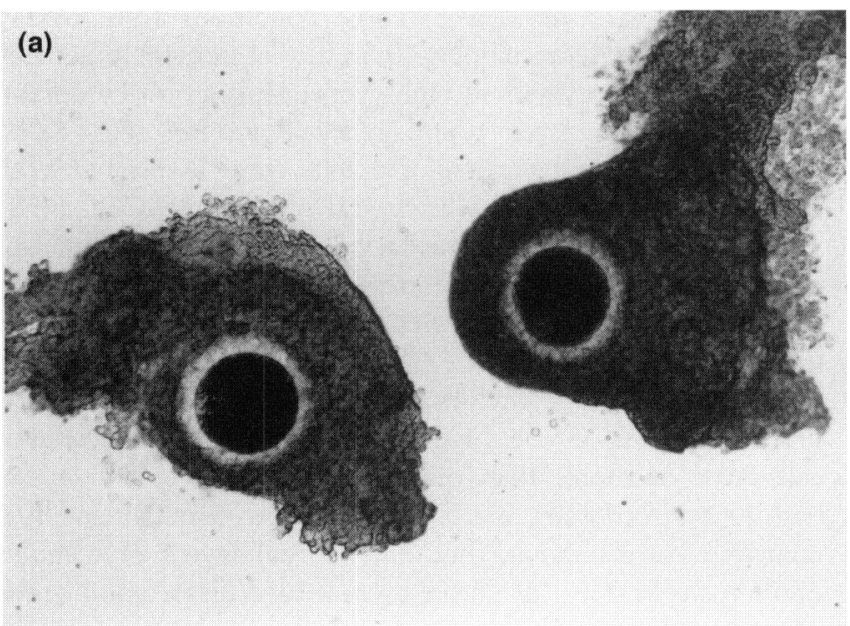

(c)

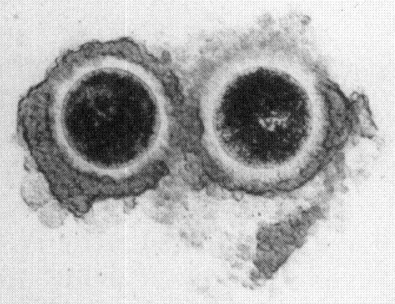

(b)

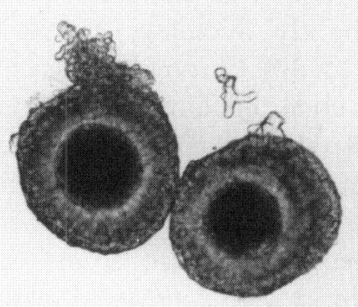

(d)

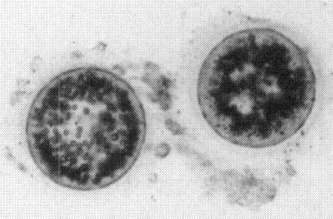

Fig. 1. Grades of various cumulus-oocyte complexes harvested from freshly excised domestic cat ovaries. (a) Grade I, excellent: oocytes have a uniform, dark cytoplasm with spherical eccentric nuclei combined with a full complement of five or more layers of tightly compacted cumulus oophorus cells. (b) Grade II, good: oocytes have a uniform, dark cytoplasm with complete complements of corona radiata cells, but fewer than five layers of supporting cumulus oophorus cells. (c) Grade III, fair: oocytes lack uniformity which is expressed as mosaic transparency of the cytoplasm; oocytes have nearly a full complement of corona radiata and some cumulus oophorus cells, but are not as tightly compacted as higher grades. (d) Grade IV, poor: oocytes with severe mosaic transparency or fragmentation of the cytoplasm and sparse complements of corona radiata and cumulus oophorus cells, some nearly denuded.

Table 1. In vitro maturation and fertilization of cat oocytes recovered from graded cumulus-oocyte complexes (COCs)

\begin{tabular}{lccc}
\hline COC grade & $\begin{array}{c}\text { Total } \\
\text { number of } \\
\text { COCs }\end{array}$ & $\begin{array}{c}\text { Number } \\
\text { maturing } \\
(\%)\end{array}$ & $\begin{array}{c}\text { Number } \\
\text { fertilized } \\
(\%)\end{array}$ \\
\hline 1 (excellent) & 91 & $54(59.3 \%)^{\mathrm{a}}$ & $27(29.7 \%)^{\mathrm{a}}$ \\
2 (good) & 216 & $70(32.4 \%)^{\mathrm{b}}$ & $25(11.6 \%)^{\mathrm{b}}$ \\
3 (fair) & 178 & $39(21.9 \%)^{\mathrm{b}}$ & $9(5.1 \%)^{\mathrm{bc}}$ \\
4 (poor) & 214 & $11(5.1 \%)^{\mathrm{c}}$ & $3(1.4 \%)^{\mathrm{c}}$ \\
\hline
\end{tabular}

a,b,c,dValues with different superscripts within a column are significantly different $(P<0.05)$.

Swanson et al., 1994) and in another in vitro system (Wolfe and Wildt, 1996). By $24 \mathrm{~h}$ after fertilization, most embryos were at the two-cell stage, and by $48 \mathrm{~h}$ nearly all were eight-cell embryos. By $120 \mathrm{~h}$ after insemination, morulae predominated. Most embryos were blastocysts by day 7 after insemination with expansion occurring from day 7 to day 9 and hatching on day 10. Compared with grade I oocytes, there was a marked decline $(P<0.05)$ in the ability of oocytes from grade II COCs to cleave after insemination. Grade III and IV oocytes demonstrated a poor ability to cleave and develop in vitro $(<1 \%)$.

\section{Discussion}

From recent detailed histological assessments of 2280 domestic cat intraovarian follicle-oocyte complexes, we identified distinctive traits of the cytoplasm and cumulus cell complement of the oocyte that appear related to the quality of the oocyte (Wood et al., 1997). The present study confirmed that these quality grades could be extrapolated and used to identify four types of COCs from whole cat ovaries with the aid of a 
Table 2. Cleavage and development of in vitro matured and fertilized cat oocytes recovered from graded cumulus-oocyte complexes (COCs)

\begin{tabular}{|c|c|c|c|c|c|c|}
\hline COC grade & $\begin{array}{c}\text { Total } \\
\text { number } \\
\text { of COCs }\end{array}$ & $\begin{array}{l}\text { Number of } \\
\text { oocytes } \\
\text { cleaving } \\
(\%)\end{array}$ & $\begin{array}{c}\text { Number of } \\
\text { morulae } \\
(\%)\end{array}$ & $\begin{array}{c}\text { Number of } \\
\text { blastocysts } \\
(\%)\end{array}$ & $\begin{array}{c}\text { Number of } \\
\text { expanded } \\
\text { blastocysts } \\
(\%)\end{array}$ & $\begin{array}{c}\text { Number of } \\
\text { hatching } \\
\text { blastocysts } \\
(\%)\end{array}$ \\
\hline 1 (excellent) & 123 & $46(37.4)^{a}$ & $38(30.9)^{a}$ & $30(24.4)^{a}$ & $21(17.1)^{a}$ & $8(6.5)^{a}$ \\
\hline $2($ good $)$ & 226 & $33(14.6)^{b}$ & $22(9.7)^{\mathrm{b}}$ & $12(5.3)^{b}$ & $10(4.4)^{\mathrm{b}}$ & $5(2.2)^{\mathrm{a}}$ \\
\hline 3 (fair) & 296 & $2(0.7)^{c}$ & $1(0.3)^{c}$ & - & - & - \\
\hline 4 (poor) & 395 & $3(0.8)^{c}$ & $1(0.3)^{c}$ & - & - & - \\
\hline
\end{tabular}

${ }^{a, b, c}$ Values with different superscripts within a column are significantly different $(P<0.05)$.

stereomicroscope. Most importantly, oocytes representing these different grades varied functionally in vitro in terms of ability to achieve nuclear maturation, fertilization and advanced embryo development. Most surprising was the small number of these high quality cat oocytes that were capable of maturing and undergoing embryo development in vitro. Of the 1849 oocytes examined in the combined experiments, only 238 $(12.9 \%)$ met the optimum criteria. In contrast, more than 1100 COCs $(61.2 \%)$ that were scored as grade III or IV had little chance of being fertilized or undergoing embryo development in vitro. None the less, if $\mathrm{COCs}$ met the highest quality score (grade I), a high percentage of oocytes cleaved following insemination, and the proportion achieving growth to morula and blastocyst stages was comparable to those observed within the oviduct and uterine horn 5-6 days after mating (Swanson et al., 1994).

Using a less selective approach for choosing oocytes (two grades), we recently reported that about $36 \%$ of intraovarian cat oocytes that were cultured for $24 \mathrm{~h}$ and then inseminated would reach the morula stage, with about $9 \%$ advancing to the blastocyst stage (Wolfe and Wildt, 1996). In Expt 2, the proportion of embryos growing to blastocysts was increased by another 15\% over the previous study, probably because the grading system was more refined. Cleaved grade II COCs developed to the blastocyst stage less frequently than grade I COCs; $30 / 46$ (65.2\%) cleaved grade I oocytes became blastocysts compared with 12/33 (36.4\%) grade II oocytes. Therefore, although the ability to undergo cleavage appears to be related to a subsequent capacity to grow in vitro, some of these lower scored oocytes remained partially compromised in both functional and developmental ability.

The most significant trait for discriminating oocyte quality in cats and its subsequent ability to be fertilized, cleave and develop in vitro appears to be cytoplasmic quality, reflected as translucent or mosaic patterns under macroscopic observation. In this respect, the cat is quite similar to the cow, because oocyte grading systems have revealed a similar cast to bovine oocyte cytoplasm and confirmation that these transformations are related to apoptosis and degenerative atresia (Kruip and Dieleman, 1982; deLoos et al., 1989, 1991; Assey et al., 1994; Long et al., 1994). Observed changes are associated with organelle-free areas of the cytoplasm as well as fewer cumulus cell-process endings penetrating the oocyte and fewer gap junctions, which explain the translucency and mosaicism. In turn, the latter observations may account for the sparse cumulus complements noted on COCs that were categorized as grade IV in this study.

In previous descriptions of sperm-oocyte maturation and interaction in cats, close similarities to the bovine model were not observed (Wildt, 1991). However, these most recent IVM/IVF findings do suggest some similarities between the two species, particularly given that most cattle studies rely on a random source of ovaries and a maturation period of $24 \mathrm{~h}$, as in domestic cats. Although many investigators have produced blastocyst-stage cattle embryos with a wide array of media and culture conditions (see review, Trounson et al., 1994), most use complex media and fetal calf serum (FCS) for best results. However, FCS is highly inhibitory to maturation of cat oocytes cultured in vitro (Wood et al., 1995). Nevertheless, one recently reported bovine culture system found that about $40 \%$ of oocytes matured in BSA-supplemented medium grow to blastocysts (Trounson et al., 1994), a result similar to that achieved in the present study for cat oocytes from grade I COCs. It also appears useful in both the bovine and domestic cat system to rely on the condition of the cumulus cell complement for accurately scoring quality of recovered oocytes. Bovine oocytes collected from medium-to-large follicles $(>2 \mathrm{~mm}$ in diameter) are more capable of maturing, being fertilized and developing as embryos than those recovered from $<2 \mathrm{~mm}$ follicles (Tan and Lu, 1990; Pavlok et al., 1992). This finding is attributed mainly to the finding that larger bovine follicles contain oocytes that have five or more layers of compact cumulus cells (deLoos et al., 1989; Blondin and Sirard, 1995). The highest quality cat oocytes in the present study were those with five or more layers of cumulus cells. We suspect that most grade I COCs originate from follicles larger than $2 \mathrm{~mm}$ in diameter because Graafian follicles in the cat are usually about $3 \mathrm{~mm}$ in diameter or greater (Wildt and Seager, 1980). One of the primary advantages of our recent histological study of the cat ovary was to confirm that oocyte atresia occurs in a high percentage of smaller antral follicles $(0.5-1.0 \mathrm{~mm})$, whereas the first signs of atresia in larger follicles occurs at the level of the mural granulosa rather than the oocyte itself (Wood et al., 1997). The present study indicates that there are inherent and programmed follicle/oocyte stimulation and regression factors controlling COC condition that are reflected in clear morphological differences and in a varying ability of the oocyte to undergo in vitro maturation, fertilization and development. 
In summary, our most recent series of related studies of IVM/IVF in cats allow the following conclusions to be made. Bovine serum albumin is the protein of choice for maximizing the ability of immature felid oocytes to achieve nuclear maturation and fertilization in vitro (Wood et al., 1995). The time of maturation (to telophase of meiosis I or metaphase of meiosis II) occurs at $24-32 \mathrm{~h}$ after initiating culture of intraovarian oocytes (Wood et al., 1995; Wolfe and Wildt, 1996). It also appears that storing whole cat ovaries in cold $\left(4^{\circ} \mathrm{C}\right)$ saline inhibits taphonomic changes (progressive post-mortem tissue degeneration), indicating that oocytes stored in this fashion have some degree of cold tolerance (Wood ef al., 1997). None the less, it is known that atresia claims the majority $(65 \%)$ of grown oocytes available for harvest from randomly collected cat ovaries (Wood et al., 1997). Despite this, oocytes recovered from ovaries stored for up to $24 \mathrm{~h}$ can mature and cleave in vitro (Wolfe and Wildt, 1996) and their developmental kinetics in culture are no different from those observed in vivo after natural mating (Swanson et al., 1994). Finally, the success of IVM/IVF in the domestic cat is directly proportional to the integrity and condition of the harvested oocyte. Oocytes with high quality cytoplasm and a cumulus cell complement have a much greater chance of maturing and being fertilized in vitro than do lower quality oocytes, and some will grow to advanced embryo stages in vitro. However, $<15 \%$ of all recovered, intraovarian oocytes could be graded as excellent, indicating that, unless alternative methods can be found to rescue low quality oocytes, rates of IVM/IVF success will continue to be limited by the finite quality of the extant COC population.

The authors thank the staff of the Petworth Animal Clinic and the Humane Society of Washington DC for contributing ovarian tissues. Support for this study was provided by a George Mason University Graduate Fellowship awarded to T. C. Wood and a grant from the Smithsonian's Scholarly Studies Program awarded to D. E. Wildt.

\section{References}

Assey RJ, Hyttel P, Greve T and Purwantara B (1994) Oocyte morphology in dominant and subordinate follicles Molecular Reproduction and Development 37 335-344

Blondin P and Sirard MA (1995) Oocyte and follicular morphology as determining characteristics for developmental competence in bovine oocytes Molecular Reproduction and Development 41 54-62

deLoos FAM, van Vliet C, van Maurik P and Kruip ThAM (1989) Morphology of immature bovine oocytes Gamete Research 24 197-204

deLoos FAM, Bevers MM, Dieleman SJ and Kruip ThAM (1991) Morphology of preovulatory bovine follicles as related to oocyte maturation Theriogenology 35 527-535

Dunn OJ (1961) Multiple comparisons among means Journal of the American Statistical Association 56 52-64

Eppig JJ and O'Brien MJ (1996) Development in vitro of mouse oocytes from primordial follicles Biology of Reproduction 54 197-207

Goodrowe KL, Howard JG, Schmidt PM and Wildt DE (1989) Reproductive biology of the domestic cat with special reference to endocrinology, sperm function and in vitro fertilization Journal of Reproduction and Fertility Supplement 39 73-90

Goodrowe KL, Hay M and King WA (1991) Nuclear maturation of domestic cat ovarian oocytes in vitro. Biology of Reproduction 45 466-470

Greulich WW (1934) Artificially induced ovulation in the cat (Felis domestica) Anatomical Record 58 217-224

Harper KM and Brackett BG (1993) Bovine blastocyst development after follicle-stimulating hormone and platelet-derived growth factor treatment for oocyte maturation in vitro. Zygote 1 27-34
Johnston LA, O'Brien SJ and Wildt DE (1989) In vitro maturation and fertilization of domestic cat follicular oocytes Gamete Research 24 434-356

Johnston LA, Donoghue AM, O'Brien SJ and Wildt DE (1991a) Culture medium and protein source influence in vitro fertilization and embryo development in the domestic cat Journal of Experimental Zoology 257 350-359

Johnston LA, Donoghue AM, O'Brien SJ and Wildt DE (1991b) Influence of temperature and gas atmosphere on in vitro fertilization and embryo development in the domestic cat journal of Reproduction and Fertility 92 $377-382$

Johnston LA, Donoghue AM, O'Brien SJ and Wildt DE (1991c) Rescue and maturation in vitro of follicular oocytes of nondomestic felid species Biology of Reproduction 45 898-906

Johnston LA, Donoghue AM, O'Brien SJ and Wildt DE (1993) Influence of culture medium and protein supplementation on in vitro oocyte maturation and fertilization in the domestic cat Theriogenology 80 829-839

Kruip ThAM and Dieleman SJ (1982) Macroscopic classification of bovine follicles and its validation by micromorphological and steroid biochemical procedures Reproduction Nutrition Developpement 22 465-473

Lonergan P, Monaghan P, Rizos D, Boland MP and Gordon I (1994) Effect of follicle size on bovine oocyte quality and developmental competence following maturation, fertilization and culture in vitro. Molecular Reproduction and Development 37 48-53

Long CR, Damiani P, Pinto-Correia C, MacLean RA, Duby RT and Robl JM (1994) Morphology and subsequent development in culture of bovine oocytes matured in vitro under various conditions of fertilization Journal of Reproducfion and Fertility 102 361-369

Luvoni GC and Oliva O (1993) Effect of medium-199 and fetal calf serum on in vitro maturation of domestic cat oocytes Journal of Reproduction and Fertility Supplement 47 203-207

Pavlok A, Lucas-Hahn A and Niemann A (1992) Fertilization and developmental competence of bovine oocytes derived from different categories of antral follicles Molecular Reproduction and Development 31 63-67

Pope CE, McRae MA, Plair BL and Dresser BL (1994) Blastocyst development in vitro following in vitro maturation, fertilization and culture of domestic cat oocytes Biology of Reproduction 50179 (Abstract)

Pope CE, McRae MA, Plair BL, Keller GL and Dresser BL (1995) In vitro and in vivo development of embryos produced by in vitro maturation (IVM) and in vitro fertilization (IVF) of domestic cat oocytes Biology Reproduction Supplement 152127 (Abstract)

Roth TL, Swanson WF and Wildt DE (1994) Developmental competence of domestic cat embryos fertilized in vivo versus in vitro. Biology of Reproduction $51441-4.51$

Swanson WF, Roth TL and Wildt DE (1994) In vivo embryogenesis, embryo migration and embryonic mortality in the domestic cat Biology of Reproduction 51 452-464

Tan SJ and Lu KH (1990) Effects of different oestrous cycle stages of ovaries and sizes of follicles on generation of IVF early embryos Theriogenology 33 335 (Abstract)

Trounson A, Pushett D, Maclellan LJ, Lewis K and Gardner DK (1994) Current status of IVM/IVF and embryo culture in humans and farm animals Theriogenology 41 57-66

Wildt DE (1991) Fertilization in cats. In A Comparative Overview of Mammalian Fertilization pp 299-328 Eds BS Dunbar and M. O'Rand. Plenum Publishing Corporation, Philadelphia

Wildt DE and Seager SWJ (1980) Ovarian and uterine morphology during the reproductive cycle (of the cat). In Current Therapy in Theriogenology pp 828-832 Ed D Morrow. WB Saunders Corporation, Philadelphia

Wildt DE, Seager SWJ and Chakraborty PK (1980) Effect of copulatory stimuli on incidence of ovulation and serum luteinizing hormone in the cat Endocrinology 107 1212-1217

Wolfe BA and Wildt DE (1996) Development to blastocysts of domestic cat oocytes matured and fertilized in vitro after prolonged cold storage Journal of Reproduction and Fertility 106 135-141

Wood TC, Byers AP, Jennette BE and Wildt DE (1995) Influence of protein and hormone supplementation on in vitro maturation and fertilization of domestic cat eggs Journal of Reproduction and Fertility 104 315-323

Wood TC, Montali RJ and Wildt DE (1997) Follicle-oocyte atresia and temporal taphonomy in cold-stored domestic cat ovaries Molecular Reproduction and Development 46 190-200 